Published in final edited form as:

Nat Nanotechnol. 2014 ; 9(12): 1054-1062. doi:10.1038/nnano.2014.274.

\title{
Squalenoyl Adenosine Nanoparticles provide Neuroprotection after Stroke and Spinal Cord Injury
}

\author{
Alice Gaudin ${ }^{1}$, Müge Yemisci ${ }^{2}$, Hakan Eroglu ${ }^{3}$, Sinda Lepêtre-Mouelhi ${ }^{1}$, Omer Faruk \\ Turkoglu ${ }^{4}$, Buket Dönmez-Demir ${ }^{2}$, Seçil Caban ${ }^{3}$, Mustafa Fevzi Sargon ${ }^{5}$, Sébastien Garcia- \\ Argote $^{6}$, Grégory Pieters ${ }^{6}$, Olivier Loreau ${ }^{6}$, Bernard Rousseau ${ }^{6}$, Oya Tagit ${ }^{7}$, Niko \\ Hildebrandt ${ }^{7}$, Yannick Le Dantec ${ }^{8}$, Julie Mougin ${ }^{1}$, Sabrina Valetti ${ }^{1}$, Hélène Chacun ${ }^{1}$, Valérie \\ Nicolas $^{9}$, Didier Desmaële ${ }^{1}$, Karine Andrieux ${ }^{*}, 1$, Yilmaz Capan ${ }^{3}$, Turgay Dalkara ${ }^{2}$, and \\ Patrick Couvreur ${ }^{*}, 1$ \\ ${ }^{1}$ Institut Galien Paris-Sud UMR CNRS 8612, Faculty of Pharmacy, University of Paris-Sud XI, \\ 92296 Châtenay-Malabry, France \\ ${ }^{2}$ Institute of Neurological Sciences and Psychiatry, Hacettepe University, Ankara 06100, Turkey \\ ${ }^{3}$ Department of Pharmaceutical Technology, Faculty of Pharmacy, Hacettepe University, Ankara \\ 06100, Turkey \\ ${ }^{4}$ Department of Neurosurgery, Ankara Ataturk Research \& Education Hospital, 06800 Bilkent \\ Ankara, Turkey \\ ${ }^{5}$ Department of Anatomy, Faculty of Medicine, Hacettepe University, Ankara 06100, Turkey \\ ${ }^{6}$ CEA Saclay, iBiTecS-S/SCBM, Labex LERMIT, 91191 Gif-sur-Yvette, France \\ ${ }^{7}$ NanoBioPhotonics, Institut d'Electronique Fondamentale, University of Paris-Sud XI, 91405, \\ Orsay Cedex, France \\ ${ }^{8}$ EA3544, Faculty of Pharmacy, University of Paris-Sud XI, 92296 Châtenay-Malabry, France \\ 9Institut d'Innovation Thérapeutique, IFR141 ITFM, Faculty of Pharmacy, University of Paris-Sud \\ XI, 92296 Châtenay-Malabry, France
}

Users may view, print, copy, and download text and data-mine the content in such documents, for the purposes of academic research, subject always to the full Conditions of use:http://www.nature.com/authors/editorial_policies/license.html\#terms

*Correspondence and requests for materials should be adressed to P.C. and K.A. patrick.couvreur@u-psud.fr,

karine.andrieux@parisdescartes.fr.

Author contributions

P.C. and T.D. conceived and designed the research. A.G. designed and performed the nanoparticles preparation, the side-effects and toxicity experiments, the stability and in vivo pharmacokinetic/biodistribution studies and the in vitro experiments. S.L. developed and performed the SQAd synthesis, D.D. helped analysing the chemical results. B.R., S.G.A., G.P. and O.L. developed and performed the radiolabelled compound synthesis. T.D. and M.Y. designed and performed the cerebral ischaemia experiments. B.D.-D. performed the histological stainings and countings for cerebral ischaemia experiments. S.C. and Y.C. were in charge of the nanoparticles preparation for the cerebral ischaemia experiments. H.E., O.F.T. and A.G. designed and performed the spinal cord injury experiments. M.F.Z. performed the ultrastructural evaluation of the spinal cord injury experiments. A.G., O.T. and N.H designed and performed the FRET NAs experiments. Y.L.D and A.G performed the sleep cycle experiments. J.M. performed the HPLC analysis. S.V. performed the complement activation experiments. H.C. helped analysing the radioactivity data and V.N. helped analysing the confocal data. P.C., T.D., K.A., A.G. and M.Y. co-wrote the paper. All authors discussed the results and commented on the manuscript.

Additional information

The authors declare no competing financial interest. Supplementary Information and Supplementary Movie accompany this paper at www.nature.com/naturenanotechnology. Reprints and permission information is available online at http://npg.nature.com/ reprintandpermissions/. 


\section{Abstract}

There is an urgent need to develop new therapeutic approaches for the treatment of severe neurological trauma, such as stroke and spinal cord injuries. However, many drugs with potential neuropharmacological activity, like adenosine, are inefficient upon systemic administration because of their fast metabolisation and rapid clearance from the bloodstream. Here, we show that the conjugation of adenosine to the lipid squalene and the subsequent formation of nanoassemblies allow a prolonged circulation of this nucleoside, to provide neuroprotection in mouse stroke and rat spinal cord injury models. The animals receiving systemic administration of squalenoyl adenosine nanoassemblies showed a significant improvement of their neurologic deficit score in the case of cerebral ischaemia, and an early motor recovery of the hindlimbs in the case of spinal cord injury. Moreover, in vitro and in vivo studies demonstrated that the nanoassemblies were able to extend adenosine circulation and its interaction with the neurovascular unit. This paper shows, for the first time, that a hydrophilic and rapidly metabolised molecule like adenosine may become pharmacologically efficient owing to a single conjugation with the lipid squalene.

Brain diseases represent a major health concern due in part to population ageing, and to the life-long disability that they imply ${ }^{1}$. Drug delivery remains the main challenge of CNS drug development ${ }^{2}$, due to a fast metabolisation and/or rapid blood clearance of most CNS drugs, as well as usually poor diffusion through the Blood-Brain Barrier (BBB) and the BloodSpinal Cord Barrier (BSCB). Another hurdle in CNS drug development is the high degree of complexity of the human brain ${ }^{3}$ and cerebral diseases ${ }^{4}$, which often involve complex processes through the interaction of multiple mechanisms between cerebrovasculature and parenchyma ${ }^{5}$. It has been suggested that nanotechnologies may promote brain delivery and efficacy of drugs ${ }^{6,7}$, owing to an improved pharmacokinetic profile and a better neurovascular unit access. However, many of these nanodevices require substantial design and complex multifunctionalisation to achieve targeted delivery of drugs which may restrain their pharmaceutical development ${ }^{8}$. In addition, if a significant although limited BBB translocation has been demonstrated with these systems, there are only few examples of real subsequent pharmacological activity ${ }^{9}$. A typical example is adenosine, a nucleoside with potential significant beneficial activity in several severe neurological disorders ${ }^{10-13}$, which has never been used for the treatment of cerebral diseases ${ }^{14}$ because of a short plasma halflife $^{15}$, the advent of moderate side effects ${ }^{16-18}$ and its inability to cross the BBB and the $\mathrm{BSCB}^{19,20}$. We report here a very simple and easy way to use the currently unusable adenosine as a neuroprotective drug following intravenous injection. We show that the bioconjugation of adenosine with the squalene, a natural and biocompatible lipid, to form an amphiphilic prodrug led to the spontaneous formation of nanoparticles with a size of ca. 120 $\mathrm{nm}$, allowing: (i) an efficient protection from rapid metabolisation, (ii) the induction of a dramatic neuroprotective effect in both an ischaemia-reperfusion model in mice and a spinal cord injury model in rats, likely due to (iii) a prolonged drug interaction with the neurovascular unit, (iv) without triggering any side-effects nor inducing systemic toxicity. Thus, the «squalenoylation» technology, which has already been applied to the intravenous administration of anticancer and antiretroviral compounds ${ }^{21-23}$, is shown here, for the first time, to be competent for the delivery of therapeutic amounts of drugs to treat CNS injuries. 


\section{Preparation and characterisation}

As illustrated in Fig. 1a, the squalenylacetic acid has been covalently linked onto the amino group of the adenosine to form the prodrug squalenoyl adenosine (Supplementary Section II. 1.). The nanoassemblies (NAs) were prepared by nanoprecipitation of an ethanolic solution of squalenoyl adenosine (SQAd) in a 5\% aqueous dextrose solution without any added surfactant, which from a toxicological point of view is a major advantage (Supplementary Fig. 1). Since adenosine is not physically encapsulated but covalently linked to the lipid nanocarrier (i.e. squalenylacetic acid), a high drug loading of $37 \%$ was reached. The design of the SQAd prodrug was intended to protect the fragile adenosine from metabolisation, when the subsequent formation of nanoassemblies allowed obtaining an aqueous suspension suitable for intravenous administration. Transmission electron microscopy (TEM) images of SQAd NAs (Fig. 1b) showed monodisperse nanoparticles with a mean diameter of ca. 120 $\mathrm{nm}$, as also confirmed by dynamic light scattering (DLS) (Fig. 1c and Supplementary Table 1). The average surface charge of the nanoassemblies was found to be ca. $-25 \mathrm{mV}$, ensuring colloidal stability for at least one month when kept at $4^{\circ} \mathrm{C}$ (Supplementary Fig. 2). The SQAd nanoassemblies were then fluorescently labelled with CholEsteryl BODIPY® ${ }^{\circledR} \mathrm{FL} \mathrm{C}_{12}$ (Supplementary Fig. 3). It was observed that the entrapment of the probe inside the nanoassemblies did not significantly modify its optical properties (Supplementary Fig. 4), whereas a 1:100 (weight:weight) ratio allowed to obtain the best emission signal by avoiding any self-quenching of the fluorophore or eximer formation ${ }^{24}$ (Supplementary Fig. 5). To allow in vivo tracking of the NAs, we synthetised radiolabelled SQAd, either by proton exchange on the purine base of the adenosine to obtain SQ- ${ }^{3} \mathrm{H}-\mathrm{Ad}$ (Supplementary Fig. 6) or performing the coupling of ${ }^{14} \mathrm{C}$-squalenylacetic acid with adenosine to obtain ${ }^{14} \mathrm{C}$-SQAd (Supplementary Fig. 7). Fluorescently and radio-labelled SQAd nanoassemblies were obtained without any significant differences in size or surface charge compared to nonlabelled SQAd NAs (Supplementary Table 1).

\section{Pharmacological efficiency}

Although adenosine receptor subtypes in the brain and periphery play complicated and sometimes opposing roles in cerebral ischaemia, central administration or continuous intravenous infusion of adenosine have been shown to be protective against stroke ${ }^{25-27}$. However, side-effects offset any potential improvement in penumbral residual blood flow ${ }^{28,29}$. Hence, any modification in adenosine delivery that may alleviate adverse reactions without losing its action on cerebral microcirculation may prove to be neuroprotective. In this study, we found that intravenous bolus administration of SQAd NAs dose-dependently decreased the infarct volume in mice subjected to $2 \mathrm{~h}$ of middle cerebral artery occlusion (MCAo) and $22 \mathrm{~h}$ of reperfusion. Pre-ischaemic injection of 7.5 or 15 $\mathrm{mg} / \mathrm{kg}$ SQAd NAs (equiv. respectively to 2.75 and $5.5 \mathrm{mg} / \mathrm{kg}$ of adenosine) significantly reduced the stroke size to $31 \pm 2$ and $17 \pm 1 \mathrm{~mm}^{3}$ respectively, compared to the control groups treated with dextrose $5 \%\left(49 \pm 1 \mathrm{~mm}^{3}\right)$, free adenosine $\left(5.5 \mathrm{mg} / \mathrm{kg}, 55 \pm 3 \mathrm{~mm}^{3}\right)$ or with unconjugated SQ NAs $\left(9.45 \mathrm{mg} / \mathrm{kg}, 44 \pm 2 \mathrm{~mm}^{3}\right.$ ) (Fig. 2a). Considering the extremely short plasma half-life of adenosine, we tested whether longer-lasting adenosine receptor agonists could exert neuroprotection comparable to NAs. Systemic administration of $\mathrm{A}_{2}$ receptor agonist (CGS21680) was not effective, whereas mice treated with $\mathrm{A}_{1}$ receptor 
agonist (CCPA) did not live for $24 \mathrm{~h}$ because of cardiac adverse effects (Supplementary

Section I.4.). To test if the neuroprotective effect observed with pre-ischaemic administration of SQAd NAs may have clinical utility, SQAd NAs $(15 \mathrm{mg} / \mathrm{kg})$ were also administered at reperfusion, $2 \mathrm{~h}$ after stroke. The infarct volume $\left(23 \pm 2 \mathrm{~mm}^{3}\right)$ was significantly smaller compared to the controls (Fig. 2a). To evaluate if this neuroprotective effect was preserved in the absence of reperfusion, a common clinical setting ${ }^{30}$, mice were subjected to $24 \mathrm{~h}$ of permanent MCAo. SQAd NAs ( $15 \mathrm{mg} / \mathrm{kg}$ ) administered $2 \mathrm{~h}$ after the induction of ischaemia significantly decreased the infarct volume $\left(24 \pm 4 \mathrm{~mm}^{3}\right)$ compared to the vehicle-treated control group $\left(54 \pm 3 \mathrm{~mm}^{3}\right.$ ) (Fig. 2a). Improvements in neurologic deficit scores paralleled the reductions in infarct size in all groups (Fig. 2c). Similar to the decrease in infarct volume (64\%), the cell death processes were also slowed down in the ischaemic areas destined to infarct in the SQAd NAs treated group ( $15 \mathrm{mg} / \mathrm{kg}$, pre-ischaemia), as shown by significant reductions at $24 \mathrm{~h}$ in the number of cells exhibiting apoptotic nuclei with clumped chromatin (Supplementary Fig. 8) or caspase-3 activation (Supplementary Fig. 9). Given the vasodilatory properties of adenosine, we investigated if the protective action of SQAd NAs could result from a primary vascular mechanism leading to a secondary parenchymal neuroprotection. We observed that most of the ischemic microvessels were patent $6 \mathrm{~h}$ after re-opening of the MCA in SQAd NAs administered mice in contrast to untreated animals in whom ischaemic capillaries were still clogged with trapped erythrocytes (Fig. 2d-e and Supplementary Fig. 10), possibly due to ischaemia-induced pericyte contractions as previously reported ${ }^{31}$. In addition, astrocyte endfeet around microvessels and endothelial nuclei were not swollen in treated animals unlike untreated mice (Fig. 2f-g). The infarct area was significantly reduced in SQAd NAs treated mice compared to the untreated controls after $6 \mathrm{~h}$ of reperfusion (Supplementary Fig. 11), that is before significant activation of inflammatory and astrogliotic mechanisms. These findings indicate that adenosine dissociated from NAs penetrating into the endothelium may interact with its receptors on pericytes of the cerebral microvessels and astrocytes endfeet, then improving microcirculation ${ }^{32}$. Moreover, adenosine has been shown to be cytoprotective in low intracellular ATP states by promoting ATP generation independently of adenosine receptors, which may have prevented the observed swelling in the endothelium and astrocyte endfeet ${ }^{33}$. Noteworthy, the concentration of adenosine attained within the capillary wall, around the neurovascular unit appears to be sufficient to mitigate microcirculation, without inducing systemic vasodilation and resulting hypotension (Supplementary Table 2). In the case of permanent MCAo, adenosine may promote the microcirculatory perfusion in periphery of the MCA area supported by collateral flow with a similar mechanism.

The promising results obtained in the cerebral ischaemia model have triggered our curiosity to further extend the pharmacological application of SQAd NAs to the treatment of the traumatic injury of the spinal cord. It has been shown that adenosine could provide neuroprotection after spinal trauma, especially through peripheral and central effects on its $\mathrm{A}_{2 \mathrm{~A}}$ receptors ${ }^{34,35}$. To determine whether the SQAd NAs could improve functional outcome in a spinal cord injury (SCI) model, we performed a behavioural and ultrastructural study in Sprague-Dawley rats undergoing a T9 contusion injury, which is the most relevant model of human $\mathrm{SCI}^{36}$. Within 5 min post-injury, we injected intravenously either SQAd NAs (32 mg/kg, i.e. equivalent dose $11.5 \mathrm{mg} / \mathrm{kg}$ adenosine and $20.2 \mathrm{mg} / \mathrm{kg}$ squalene), SQ 
NAs $(20.5 \mathrm{mg} / \mathrm{kg})$, free adenosine $(11.5 \mathrm{mg} / \mathrm{kg})$ or a solution of dextrose $5 \%$ as control. Functional evaluations were performed ${ }^{37}$ at 24,48 and $72 \mathrm{~h}$ post-injury, and then regularly up to 28 days post-injury. The scores of animals in all groups are presented in the Fig. $3 \mathrm{~b}$ and Supplementary Table 3, and illustrated in the movie available in the web version of this article. $72 \mathrm{~h}$ after trauma, animals injected post-injury with either dextrose 5\% solution or free adenosine solution (Supplementary Fig. 12a,b and Supplementary Movie) were unable to voluntary move their hindlimbs, in accordance with the presence of an important traumatic area observed on the cords (Fig. 3c-d). At opposite, animals injected with the SQAd NAs did not present any visible traumatic area on their cords (Fig. 3e), and were fully able to walk with consistent coordination between the hindlimbs and forelimbs (Supplementary Fig. 12c-d-e-f and Supplementary Movie). As soon as 9 days after trauma, SQAd NAs treated animals reached a score of 14.4 (Fig. 3b), which is widely accepted as promising and clinically meaningful ${ }^{36}$ as it is a sign of axonal transduction through the lesion site ${ }^{38}$. All other treatments group did not exceed a score of 5 until 28 days posttrauma (Fig. 3b). Since it has been shown that the preservation of only 5 to $10 \%$ of axons in each individual tract was sufficient to achieve meaningful locomotor recovery ${ }^{1}$, we further investigated the ultrastructure of the myelinated axons 2 and 28 days post-injury, using transmission electron microscopy (Fig. 3f-i). The examination of the white matter of control animals (SQ NAs or free adenosine solution) revealed that the small, medium and large sized myelinated axons had interruptions in myelin configuration (Fig. 3g-h). In the case of the animals treated with the SQAd NAs, axons with separations in myelin configuration was detected but most of these neurons were medium and large sized, when a few of the medium sized and most of the small sized myelinated axons were normal (Fig. 3i). The quantification of these observations showed that the SQAd NAs treatment significantly decreased the damages to the small (Fig. 3j) and medium (Supplementary Fig. 13) sized myelinated axons and the swelling of mitochondria (Supplementary Fig. 14), as soon as 2 days post-trauma.

\section{Side effects and systemic toxicity}

The emergence of side effects is an important limitation for the use of most CNS medicines ${ }^{39}$. As adenosine is known to be a neuromodulator involved in numerous neurological processes ${ }^{13}$, its therapeutic use may be associated with the appearance of side effects such as loss of appetite ${ }^{17,40}$ or alteration of sleep cycle ${ }^{18,41}$. We monitored the effect of SQAd NAs on food intake and body weight. The SQAd NAs intravenous administration $(15 \mathrm{mg} / \mathrm{kg}$ ) did not induce any modification neither in the daily food intake behaviour (Fig. 4a), nor in the weight variations (Supplementary Fig. 15) of the animals, compared to the controls (dextrose 5\%). The first nocturnal food intake following the injection of the treated animals was not significantly modified (Supplementary Fig. 16) showing no acute effect of the NAs. As for the possible impact of SQAd NAs on sleep cycle it was observed that the administration of SQAd NAs did not modify neither the sleep quantities of Non Rapid Eye Movement (NREM) sleep (Fig. 4b, Supplementary Fig. 17a) and Rapid Eye Movement (REM) sleep (Supplementary Fig. 17b), nor the wake quantity (Supplementary Fig. 18) of the treated mice compared to the controls. As accumulation of adenosine in the extracellular space of basal latencies to NREM and REM sleeps. It was observed that SQAd NAs administration did not significantly modify neither these latencies (Fig. 4c), nor the 
electroencephalogram power spectra (Supplementary Table 4). These results confirm that the dramatic pharmacological efficiency of SQAd NAs was obtained without triggering any side effect commonly associated with central administration of adenosine.

We then investigated the overall toxicity of intravenously administered SQAd NAs (15 $\mathrm{mg} / \mathrm{kg}, 1,7$ and 28 days post-injection) to mice, compared to control animals (dextrose 5\%). No differences were noted between both groups in terms of white blood cells count (Fig. 4d), red blood cells count (Fig. 4e), and other haematological parameters (Supplementary Fig. 19). The levels of aspartate aminotransferase (Fig. 4f), alanine aminotransferase (Supplementary Fig. 20a), creatinine (Fig. 4g) and urea (Supplementary Fig. 20b) were not increased in the SQAd NAs group, indicating no damage to the kidneys or the liver. The observations of kidneys, liver and spleen showed no morphological damages after SQAd NAs administration, $24 \mathrm{~h}$ and 28 days post-injection (Fig. 4h-m). We did not observe any nanoassemblies in the pulmonary territory showing no signs of alveolar obstruction or inflammation (Fig. 4n-o). Finally, using 2D immunoelectrophoresis we showed that the SQAd NAs led to only a slight complement activation in human plasma (Supplementary Fig. 21). Altogether, these results show that the SQAd NAs may be considered as safe upon systemic intravenous administration at the therapeutic dose of $15 \mathrm{mg} / \mathrm{kg}$.

\section{Plasma reservoir effect}

We further investigated the mechanism behind the observed pharmacological activity of SQAd NAs by performing biodistribution studies using two types of radiolabelled NAs: one with a ${ }^{14} \mathrm{C}$ labelling located on the squalene moiety and the other with a ${ }^{3} \mathrm{H}$ labelling on the adenosine moiety (Supplementary Sections I.2. and I.3.). Concerning the brain, while ${ }^{3} \mathrm{H}$ could be detected, lack of ${ }^{14} \mathrm{C}$ clearly demonstrated the absence of nanoparticles penetration (Supplementary Fig. 22). Additional assays using High Performance Liquid Chromatography with radioactive detection (radio-HPLC) also confirmed the absence of either SQAd or adenosine in the brain after injection of ${ }^{3} \mathrm{H}$ labelled SQAd $(15 \mathrm{mg} / \mathrm{kg})$ (Supplementary Fig. 23b). As expected, adenosine was not detected in the brain $1 \mathrm{~h}$ following administration of free ${ }^{3} \mathrm{H}$-Adenosine (Supplementary Fig. 23c). These data clearly show that the SQAd NAs efficacy could not be explained by a central pharmacological activity, but rather by a primary vascular mechanism leading to a secondary parenchymal neuroprotection as already indicated by the improved microcirculation in the stroke study (Fig. 2d, e). This also suggested that the effectiveness of SQAd NAs in both spinal cord injury and brain ischaemia could result from a multi-targeted pleiotropic action ${ }^{5}$ on the cells of the neurovascular unit or other peripheral cell types ${ }^{34,43}$ thanks to a sustained systemic circulation. Therefore, the colloidal stability of the SQAd NAs was first analysed in mouse plasma. Apart from a slight increase likely due to the formation of a protein corona at their surface, the mean size of the NAs did not vary (Fig. 5a, Supplementary Fig. 24) suggesting a good colloidal stability in the peripheral compartment for at least two hours. The chemical stability of SQAd was further explored after incubation in mouse plasma, and it was clearly observed that SQAd NAs provided a sustained protection of the prodrug toward enzymatic degradation (Fig. 5b). Finally, the stability of SQ- ${ }^{3} \mathrm{H}-\mathrm{Ad}$ after intravenous administration of the NAs was assessed by radio-HPLC. We observed that a significant amount of SQAd remained intact in the blood for as long as $1 \mathrm{~h}$ post-injection (Fig. 5c and Supplementary 
Fig. 25 and 26) whereas nothing was detected already 1 min post-injection of ${ }^{3} \mathrm{H}$-Adenosine free (Supplementary Fig. 27).

As a whole, these data showed that the linkage of adenosine to squalene and the assembly of the bioconjugate as nanoparticles provided a dramatic protection of adenosine from metabolisation both in vitro after incubation with mouse plasma, and in vivo after intravenous injection. Thus, SQAd NAs represent a reservoir of adenosine in the bloodstream, which could facilitate delivery to endothelial cells and further cerebrovascular protection. Therefore, the ability of SQAd nanoassemblies to interact with endothelial cells was investigated in vitro using the hCMEC/D3 human cerebral microvascular endothelial cells mode ${ }^{44}$. Using flow cytometry and confocal microscopy, we observed that those cells were able to internalise the SQAd NAs (Fig. 6a-h; Supplementary Fig. 28), as it was also observed using radiolabelled NAs (Supplementary Fig. 29). By labelling SQAd NAs with two fluorophores displaying Förster Resonance Energy Transfer (FRET) (Supplementary Section I.4.), it was possible to distinguish between intact NAs (presence of a FRET signal) and disassembled NAs (decrease of the FRET signal) after cell capture. Quantification of the FRET signal by flow cytometry showed that SQAd NAs were taken intact by the endothelial cells during the first $6 \mathrm{~h}$ of incubation (Fig. 6i), before NAs progressively dissociated over time inside the cells, confirming previous in vivo observations (Supplementary Fig. 23) showing that intact SQAd NAs were unable to enter the CNS.

In this communication, we show for the first time that the linkage of adenosine to squalene and the subsequent construction as nanoassemblies allow the efficient administration of this molecule with significant pharmacological activity in a brain ischaemia and a spinal cord injury models. The demonstrated plasma reservoir effect of SQAd NAs allowed improvement of microcirculation leading to a secondary parenchyma neuroprotection. Although further studies are needed to more precisely describe the exact therapeutic mechanism and to determine how dosage, administration frequency and timing of treatment with SQAd NAs may affect the clinical outcome, this study opens a new exciting perspective for the treatment of severe neurological diseases where tissue ischaemia and/or trauma are involved.

\section{Methods}

\section{SQAd nanoassemblies}

SQAd nanoassemblies were prepared using the nanoprecipitation technique. Briefly, SQAd was dissolved in absolute ethanol, and added dropwise under strong stearing to a $5 \%(\mathrm{w} / \mathrm{v})$ dextrose solution. Ethanol was then completely evaporated to obtain an aqueous suspension of pure SQAd nanoassemblies. Fluorescent nanoassemblies were obtained by the same procedure, unless $1 \%(\mathrm{w} / \mathrm{w})$ of the fluorescent probe CholEsteryl BODIPY® $\mathrm{FL} \mathrm{C}_{12}$ was added to the ethanolic phase. Similarly, radiolabelled nanoassemblies were obtained by adding SQ-- ${ }^{3} \mathrm{H}-\mathrm{Ad}$ or ${ }^{14} \mathrm{C}-\mathrm{SQAd}$ to the ethanolic SQAd solution. Nanoassemblies size and surface charge were systematically measured after preparation using a Malvern Nano ZS and morphology observed by cryogenic transmission electron microscopy. 


\section{Focal cerebral ischaemia injury model}

The proximal MCA was occluded by filament method in male SWISS albino mice (28-32 g) under isoflurane anaesthesia. To confirm occlusion and reperfusion, the regional cerebral blood flow was measured by a laser-Doppler flowmetry. The body temperature was kept at $37 \pm 0.1^{\circ} \mathrm{C}$. The tissue $\mathrm{O}_{2}$ saturation and arterial blood pressure were monitored noninvasively. Mice were randomly allocated to 10 groups and were subjected to $2 \mathrm{~h}$ ischaemia and $22 \mathrm{~h}$ of reperfusion or $24 \mathrm{~h}$ of permanent ischaemia (Supplementary Table 2 and 6). Treatments were administered intravenously just before inducing ischaemia or $2 \mathrm{~h}$ after ischaemia ( $\mathrm{N}=6$ animals/group, Supplementary Table 6). Mice were blindly neurologically evaluated before sacrificed. Infarct volumes were assessed by Nissl staining, caspase-3 activation by immunolabeling for cleaved caspase- 3 and apoptotic nuclear morphology by dark field microscopy. Another set of animals were sacrificed and perfused to flush erythrocytes, $6 \mathrm{~h}$ after $2 \mathrm{~h}$ MCAo, and entrapped erythrocytes were rendered fluorescent with $\mathrm{NaBH}_{4}$.

\section{Spinal cord injury model in rats}

After general anaesthesia using xylazine and ketamine hydrochloride, Sprague-Dawley rats (200-250 g) underwent a laminectomy and spinal cord injury was produced by the drop of a $4 \mathrm{~g}$ weight from $12.5 \mathrm{~mm}$ height. Within $5 \mathrm{~min}$ post-injury, treatments were injected intravenously ( $\mathrm{N}=10$ animals/group, Supplementary Table 8). Functional evaluations were performed blindly 24,48 and $72 \mathrm{~h}$ and regularly up to 28 days post-injury using the Basso, Beattie and Bresnahan grading scale. For ultrastructural evaluation, tissue samples were resected from the traumatic area of the spine $48 \mathrm{~h}$ and 28 days post-injury. Tissue samples were examined blindly by transmission electron microscopy (TEM).

\section{Assessment of the effect on food intake and sleep cycle of the SQAd NAs in mice}

$25 \mathrm{~g}$ male SWISS albino mice housed in individual cages were injected with either SQAd NAs $(15 \mathrm{mg} / \mathrm{kg}$ ) or dextrose $5 \%$ ( $\mathrm{N}=10$ animals/group). Individual mice and their corresponding food were weighed 12 hours post-injection and then regularly for seven days. For sleep cycle experiments, $25 \mathrm{~g}$ male SWISS albino mice were implanted with two cortical, two ocular and two muscular electrodes. 7 days post-surgery, they were individually placed in recording cages and allowed to habituate for three days. On the day of the experiment, the mice were disconnected, intravenously injected with either SQAd NAs (15 $\mathrm{mg} / \mathrm{kg}$ ) or dextrose $5 \%(\mathrm{~N}=15$ animals/group) and then immediately reconnected for $24 \mathrm{~h}$ recording.

\section{Assessment of the systemic toxicity of the SQAd NAs in mice}

$25 \mathrm{~g}$ male SWISS albino mice were injected with either SQAd NAs $(15 \mathrm{mg} / \mathrm{kg})$ or dextrose $5 \%(\mathrm{~N}=5$ animals/group). 1,7 or 28 days post-injection, the animals were anaesthetised using pentobarbital and blood was collected by intracardiac sampling for hematology and serum analysis. Liver, kidneys, spleen and lungs were then excised, fixed, paraffinembedded, and cut into $5 \mu \mathrm{m}$-thick sections. HES staining was performed on all the organs for analysis of the morphology. 


\section{Pharmacokinetic of the radiolabelled SQAd NAs in mice}

Radiolabelled SQAd NAs were injected to $25 \mathrm{~g}$ male SWISS albino mice $(15 \mathrm{mg} / \mathrm{kg}, 600$ $\mu \mathrm{Ci} / \mathrm{kg}$ ). $1 \mathrm{~min}, 5 \mathrm{~min}, 15 \mathrm{~min}, 30 \mathrm{~min}, 1 \mathrm{~h}$ and $24 \mathrm{~h}$ post-injection animals were anaesthetised using pentobarbital, blood was collected by intracardiac sampling and immediately centrifuged to isolate plasma. Samples were then analysed by radio-HPLC.

\section{Nanoparticles internalisation by hCMEC/D3 (flow cytometry and confocal microscopy)}

hCMEC/D3 cells were seeded in 24-wells plates $\left(50,000 \mathrm{cells} / \mathrm{cm}^{2}\right)$. Cells were grown for 7 days and then incubated with $10 \mu \mathrm{g} / \mathrm{mL}$ fluorescent SQAd NAs. For flow cytometry, cell fluorescence was recorded using a flow cytometer C6 with an excitation source at $488 \mathrm{~nm}$ and collecting the emission at $515 \mathrm{~nm}$. For confocal microscopy, if required at the end of the incubation time, nuclei were stained with $10 \mu \mathrm{M}$ of SYTO61 for $2 \mathrm{~h}$ at $37^{\circ} \mathrm{C}$, followed by membranes staining with $10 \mu \mathrm{g} / \mathrm{mL}$ WGA AlexaFluor 555 for $20 \mathrm{~min}$ at $37^{\circ} \mathrm{C}$. Cells were then fixed and imaged with a LSM510 Zeiss confocal microscope using lasers at $488 \mathrm{~nm}$ (nanoassemblies), $543 \mathrm{~nm}$ (membranes) and $633 \mathrm{~nm}$ (nuclei).

\section{Supplementary Material}

Refer to Web version on PubMed Central for supplementary material.

\section{Acknowledgements}

The research leading to these results has received funding from the European Research Council allocated to Pr. Patrick Couvreur, under the European Community's Seventh Framework Programme FP7/2007-2013 (grant agreement no. 249835). Alice Gaudin is supported by a NerF-ENP fellowship provided by the Région Ile-deFrance. Pr. Turgay Dalkara's work is supported by the Turkish Academy of Sciences. Müge Yemisci is supported by a limited grant by L'Oréal, Turkey. Hakan Eroglu has been supported by the Hacettepe University Scientific Research Project (project number: 013D04301002). The authors acknowledge Dunja Sobot (Institut Galien ParisSud XI, France) for her help with flow cytometry experiments, Miss Olivia Bawa and Dr Paule Opolon (Institut Gustave Roussy, Villejuif, France) for the analysis of the morphology on stained slides, Dr Monika Wittner (Institut Gustave Roussy, Villejuif, France) for the hematology study, Dr Stéphanie Beurlet (VEBIO Lab, France) for biochemical dosages and Dr Martine Parrod (Bertin PHARMA, Orléans, France) for the radio-HPLC analysis.

\section{References}

1. Profyris $\mathrm{C}$, et al. Degenerative and regenerative mechanisms governing spinal cord injury. Neurobiol. Dis. 2004; 15:415-436. [PubMed: 15056450]

2. Pardridge WM. Non-invasive drug delivery to the human brain using endogenous blood-brain barrier transport systems. Pharm. Sci. Technolo. Today. 1999; 2:49-59. [PubMed: 10234207]

3. Palmer AM, Alavijeh MS. Translational CNS medicines research. Drug. Discov. Today. 2012; 17:1068-1078. [PubMed: 22580061]

4. Pangalos MN, Schechter LE, Hurko O. Drug development for CNS disorders: strategies for balancing risk and reducing attrition. Nat. Rev. Drug Discov. 2007; 6:521-532. [PubMed: 17599084]

5. Zhang L, Zhang ZG, Chopp M. The neurovascular unit and combination treatment strategies for stroke. Trends Pharmacol. Sci. 2012; 33:415-422. [PubMed: 22595494]

6. Andrieux K, Couvreur P. Polyalkylcyanoacrylate nanoparticles for delivery of drugs across the blood-brain barrier. Wiley Interdiscip. Rev. Nanomed. Nanobiotechnol. 2009; 1:463-474. [PubMed: 20049811]

7. Nair SB, Dileep A, Rajanikant GK. Nanotechnology based diagnostic and therapeutic strategies for neuroscience with special emphasis on ischemic stroke. Curr. Med. Chem. 2012; 19:744-756.

[PubMed: 22204347] 
8. Sun Q, Radosz M, Shen Y. Challenges in design of translational nanocarriers. J. Control. Release. 2012; 164:156-169. [PubMed: 22664472]

9. Yang H. Nanoparticle-mediated brain-specific drug delivery, imaging, and diagnosis. Pharm. Res. 2010; 27:1759-1771. [PubMed: 20593303]

10. Boison D. Adenosine as a neuromodulator in neurological diseases. Curr. Opin. Pharmacol. 2008; 8:2-7. [PubMed: 17942368]

11. de Mendonca A, Sebastiao AM, Ribeiro JA. Adenosine: does it have a neuroprotective role after all? Brain Res. Rev. 2000; 33:258-274. [PubMed: 11011069]

12. Williams-Karnesky RL, Stenzel-Poore MP. Adenosine and stroke: maximizing the therapeutic potential of adenosine as a prophylactic and acute neuroprotectant. Curr. Neuropharmacol. 2009; 7:217-227. [PubMed: 20190963]

13. Fredholm BB, Chen JF, Cunha RA, Svenningsson P, Vaugeois JM. Adenosine and brain function. Int. Rev. Neurobiol. 2005; 63:191-270. [PubMed: 15797469]

14. Gomes CV, Kaster MP, Tome AR, Agostinho PM, Cunha RA. Adenosine receptors and brain diseases: neuroprotection and neurodegeneration. Biochim. Biophys. Acta. 2011; 1808:13801399. [PubMed: 21145878]

15. Moser GH, Schrader J, Deussen A. Turnover of adenosine in plasma of human and dog blood. Am. J. Physiol. 1989; 256:C799-806. [PubMed: 2539728]

16. Cerqueira MD, Verani MS, Schwaiger M, Heo J, Iskandrian AS. Safety profile of adenosine stress perfusion imaging: results from the Adenoscan Multicenter Trial Registry. J. Am. Coll. Cardiol. 1994; 23:384-389. [PubMed: 8294691]

17. Levine AS, Morley JE. Purinergic regulation of food intake. Science. 1982; 217:77-79. [PubMed: 7046046]

18. Basheer R, Strecker RE, Thakkar MM, McCarley RW. Adenosine and sleep-wake regulation. Prog. Neurobiol. 2004; 73:379-396. [PubMed: 15313333]

19. Pardridge WM, Yoshikawa T, Kang YS, Miller LP. Blood-brain barrier transport and brain metabolism of adenosine and adenosine analogs. J. Pharmacol. Exp. Ther. 1994; 268:14-18. [PubMed: 8301550]

20. Isakovic AJ, Abbott NJ, Redzic ZB. Brain to blood efflux transport of adenosine: blood-brain barrier studies in the rat. J. Neurochem. 2004; 90:272-286. [PubMed: 15228584]

21. Couvreur P, et al. Squalenoyl nanomedicines as potential therapeutics. Nano Lett. 2006; 6:25442548. [PubMed: 17090088]

22. Reddy LH, et al. Preclinical toxicology (subacute and acute) and efficacy of a new squalenoyl gemcitabine anticancer nanomedicine. J. Pharmacol. Exp. Ther. 2008; 325:484-490. [PubMed: 18258784]

23. Hillaireau H, et al. Anti-HIV efficacy and biodistribution of nucleoside reverse transcriptase inhibitors delivered as squalenoylated prodrug nanoassemblies. Biomaterials. 2013; 34:48314838. [PubMed: 23562054]

24. Bisgaier CL, Minton LL, Essenburg AD, White A, Homan R. Use of fluorescent cholesteryl ester microemulsions in cholesteryl ester transfer protein assays. J. Lipid Res. 1993; 34:1625-1634. [PubMed: 8228645]

25. Kitagawa H, Mori A, Shimada J, Mitsumoto Y, Kikuchi T. Intracerebral adenosine infusion improves neurological outcome after transient focal ischemia in rats. Neurol. Res. 2002; 24:317323. [PubMed: 11958429]

26. Tatlisumak T, et al. Delayed treatment with an adenosine kinase inhibitor, GP683, attenuates infarct size in rats with temporary middle cerebral artery occlusion. Stroke. 1998; 29:1952-1958. [PubMed: 9731623]

27. Pignataro G, Simon RP, Boison D. Transgenic overexpression of adenosine kinase aggravates cell death in ischemia. J. Cereb. Blood Flow Metab. 2007; 27:1-5. [PubMed: 16685255]

28. von Lubitz DK. Adenosine and cerebral ischemia: therapeutic future or death of a brave concept? Eur. J. Pharmacol. 1999; 371:85-102. [PubMed: 10355598]

29. Echavarria-Pinto M, et al. Low coronary microcirculatory resistance associated with profound hypotension during intravenous adenosine infusion: implications for the functional assessment of coronary stenoses. Circ. Cardiovasc. Interv. 2014; 7:35-42. [PubMed: 24399244] 
30. Go AS, et al. Heart disease and stroke statistics--2014 update: a report from the American Heart Association. Circulation. 2014; 129:e28-e292. [PubMed: 24352519]

31. Yemisci M, et al. Pericyte contraction induced by oxidative-nitrative stress impairs capillary reflow despite successful opening of an occluded cerebral artery. Nat. Med. 2009; 15:1031-1037. [PubMed: 19718040]

32. Hamilton NB, Attwell D, Hall CN. Pericyte-mediated regulation of capillary diameter: a component of neurovascular coupling in health and disease. Front. Neuroenergetics. 2010; 2:1-14. [PubMed: 20162100]

33. Li S, et al. Intracellular ATP concentration contributes to the cytotoxic and cytoprotective effects of adenosine. PLoS One. 2013; 8:e76731. [PubMed: 24098558]

34. Paterniti I, et al. Selective adenosine A2A receptor agonists and antagonists protect against spinal cord injury through peripheral and central effects. J. Neuroinflammation. 2011; 8:31. [PubMed: 21486435]

35. Okonkwo DO, et al. A comparison of adenosine A2A agonism and methylprednisolone in attenuating neuronal damage and improving functional outcome after experimental traumatic spinal cord injury in rabbits. J. Neurosurg. Spine. 2006; 4:64-70. [PubMed: 16506468]

36. Kwon BK, Hillyer J, Tetzlaff W. Translational research in spinal cord injury: a survey of opinion from the SCI community. J. Neurotrauma. 2010; 27:21-33. [PubMed: 19751098]

37. Basso DM, Beattie MS, Bresnahan JC. Graded histological and locomotor outcomes after spinal cord contusion using the NYU weight-drop device versus transection. Exp. Neurol. 1996; 139:244-256. [PubMed: 8654527]

38. Shi Y, et al. Effective repair of traumatically injured spinal cord by nanoscale block copolymer micelles. Nat. Nanotechnol. 2010; 5:80-87. [PubMed: 19898498]

39. Alavijeh MS, Palmer AM. Measurement of the pharmacokinetics and pharmacodynamics of neuroactive compounds. Neurobiol. Dis. 2010; 37:38-47. [PubMed: 19818851]

40. Levine AS, Morley JE. Effect of intraventricular adenosine on food intake in rats. Pharmacol. Biochem. Behav. 1983; 19:23-26. [PubMed: 6684775]

41. Portas CM, Thakkar M, Rainnie DG, Greene RW, McCarley RW. Role of adenosine in behavioral state modulation: a microdialysis study in the freely moving cat. Neuroscience. 1997; 79:225-235. [PubMed: 9178878]

42. Porkka-Heiskanen T, Alanko L, Kalinchuk A, Stenberg D. Adenosine and sleep. Sleep Med. Rev. 2002; 6:321-332. [PubMed: 12531135]

43. Melani A, Corti F, Cellai L, Giuliana Vannucchi M, Pedata F. Low doses of the selective adenosine A2A receptor agonist CGS21680 are protective in a rat model of transient cerebral ischemia. Brain Res. 2014; 1551:59-72. [PubMed: 24457041]

44. Poller B, et al. The human brain endothelial cell line hCMEC/D3 as a human blood-brain barrier model for drug transport studies. J. Neurochem. 2008; 107:1358-1368. [PubMed: 19013850] 

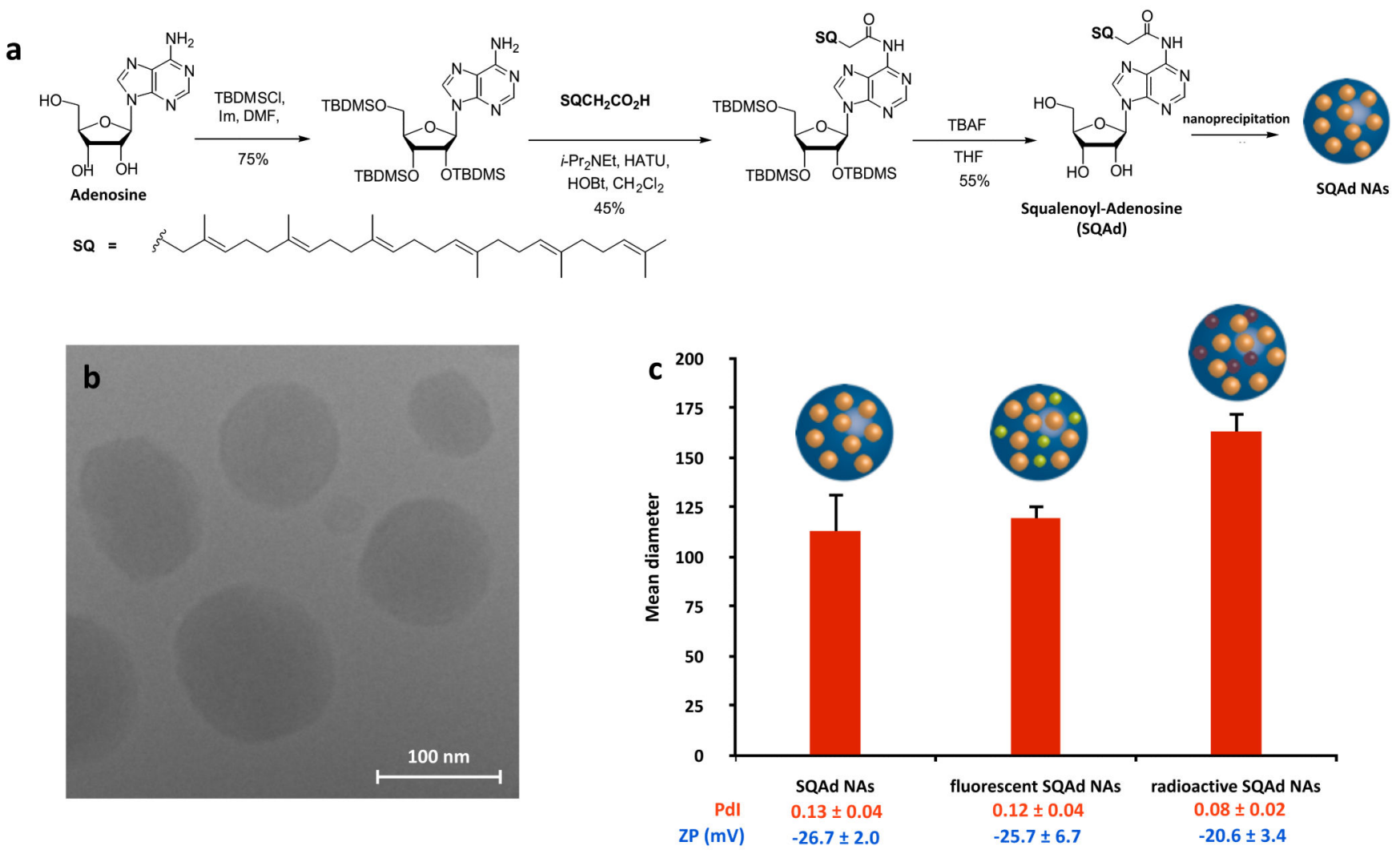

Figure 1. Preparation and characterisation of the SQAd nanoassemblies

a, Squalenoyl-Adenosine (SQAd) was prepared in a three steps synthesis and the SQAd NAs were obtained by nanoprecipitation. $\mathbf{b}$, The morphological appearance of SQAd was observed by cryoTEM. c, The size was measured by Dynamic Light Scattering (DLS). Size distributions were highly monodisperse as the Polydispersity Index (PdI) was inferior to 0.15 , and the nanoassemblies were negatively charged as shown by zeta potential (ZP) values. No modifications of the size, PdI or ZP were observed when a fluorescent probe (green sphere inside SQAd NAs) was incorporated, nor when radiolabelled NAs (NAs incorporating burgundy spheres) were prepared (data presented as mean $(\mathrm{nm}) \pm \mathrm{SD}$, Supplementary Table 1 for detailled DLS characterisation). 


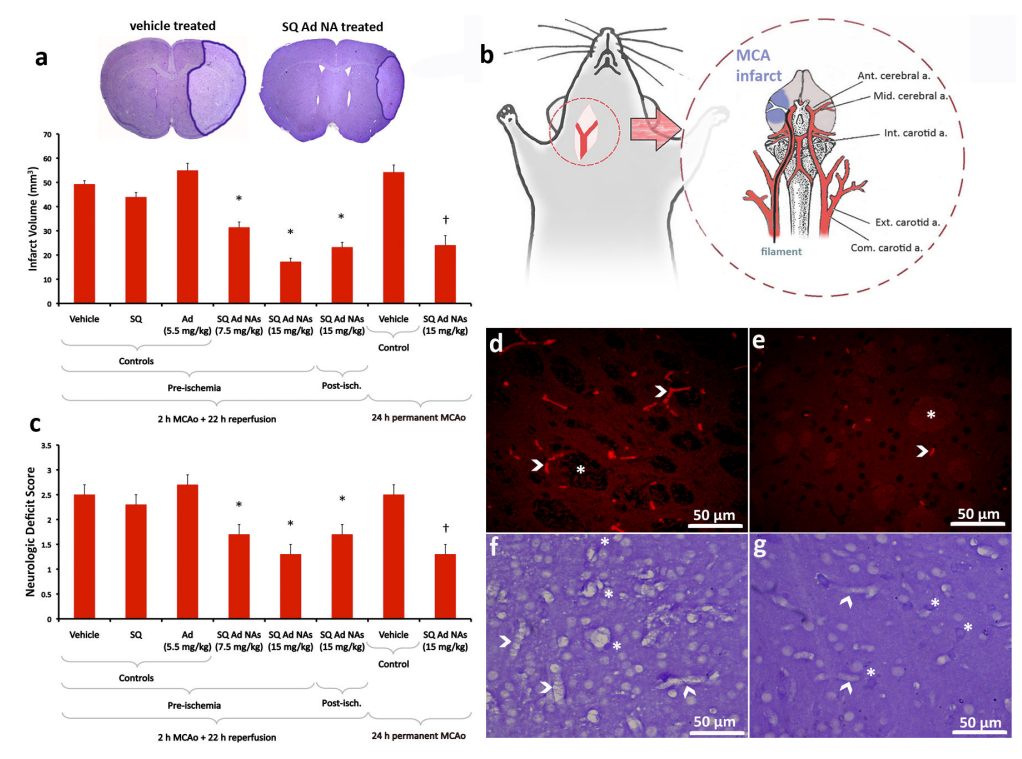

Figure 2. Systemic administration of SQAd nanoassemblies provides significant neuroprotection in a mouse model of cerebral ischaemia

a, Ischaemic volumes in control and treated mice subjected to transient ( $2 \mathrm{~h}$ MCAo and $22 \mathrm{~h}$ reperfusion) and permanent ( $24 \mathrm{~h} \mathrm{MCAo}$ ) focal cerebral ischaemia were identified by reduced Nissl staining under light microscope (magnification $\times 10$, insets) (data presented as mean $\left(\mathrm{mm}^{3}\right) \pm \mathrm{SD}, \mathrm{N}=6$ animals/group, $\dagger$ and $*$ mean $\mathrm{p}<0.05$ compared to respective controls). Intravenous administration of $7.5 \mathrm{mg} / \mathrm{kg}$ or $15 \mathrm{mg} / \mathrm{kg}$ SQAd NAs just before ischaemia or $2 \mathrm{~h}$ post-ischaemia significantly decreased the infarct volume compared to control groups that received vehicle (dextrose 5\%), adenosine-unconjugated SQ NAs (9.45 $\mathrm{mg} / \mathrm{kg}$ ) or free adenosine $(5.5 \mathrm{mg} / \mathrm{kg})$. A significant therapeutic effect was also observed when SQAd NAs were administered $2 \mathrm{~h}$ post-ischaemia in the permanent MCAo model. b, MCAo was performed in mice under anaesthesia by the intraluminal filament method. A filament was advanced in the internal carotid artery through a small incision proximal to the bifurcation up to the origin of MCA to interrupt blood flow to the MCA territory. c, The significant neuroprotective effect of SQAd NAs was also evident in neurologic evaluation (data presented as mean $\pm \mathrm{SD}, \mathrm{N}=6$ animals/group, $\dagger$ and $*$ mean $\mathrm{p}<0.05$ compared to respective controls). Neurologic deficit scores, assessed $24 \mathrm{~h}$ after the induction of stroke, paralleled the changes in the infarct volume although the decreases in deficit scores were less striking possibly because some pyramidal fibres descending from the recovered cortex could not escape damage in the ischaemic subcortical area. d,e, In untreated mice, capillaries in the ischaemic brain were filled with trapped erythrocytes (d, red fluorescence, arrowheads) $6 \mathrm{~h}$ after re-opening of the MCA following $2 \mathrm{~h}$ of occlusion, whereas majority of capillaries were not clogged in SQAd NAs treated mice (e). The treatment also reduced ischaemic tissue changes such as swelling of axon bundles $(\mathbf{e}, *)$. f,g, Similarly, capillary structure was well preserved in treated animals (g) unlike controls $(\mathbf{f})$, in which astrocyte end-feet ensheathing capillaries as well as endothelial nuclei were swollen (arrowheads) in addition to oedematous neuropil and several dark stained degenerating cells surrounded by swollen end-feet $(\mathbf{f}, *)$. In treated mice, parenchymal ischaemic changes were mild, limited to light swelling and scattered degenerating neurons $(\mathbf{g}, *)$. 

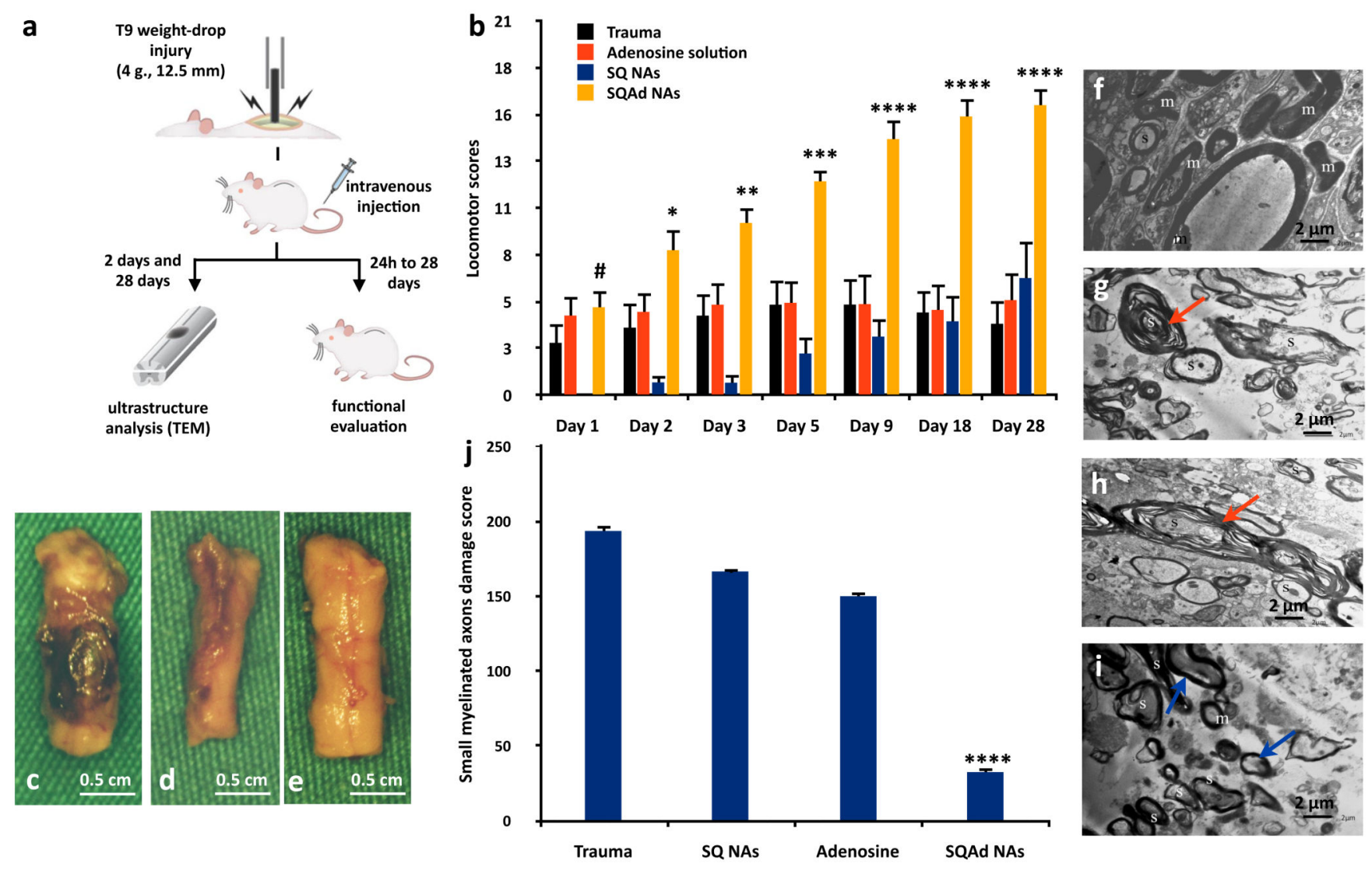

Figure 3. Pharmacological efficiency of the SQAd nanoassemblies in a model of spinal cord injury in rats

a, The pharmacological efficiency of the SQAd NAs was assessed in a T9 contusion spinal cord injury model. Within $5 \mathrm{~min}$, the animals were intravenously injected with either dextrose $5 \%$, SQAd NAs (32 mg/kg, equiv. adenosine $11.5 \mathrm{mg} / \mathrm{kg}, \mathrm{N}=10$ animals), SQ NAs $(20.2 \mathrm{mg} / \mathrm{kg}, \mathrm{N}=10$ animals) or free adenosine $(11.5 \mathrm{mg} / \mathrm{kg}, \mathrm{N}=10$ animals $)$. b, After $24 \mathrm{~h}$, $48 \mathrm{~h}$ and $72 \mathrm{~h}$, and up to 28 days post-trauma, the animals were functionally graded using the Basso, Beattie and Bresnahan grading scale (data presented as mean \pm SEM, \# ns, $* \mathrm{p}<0.05, * * \mathrm{p}<0.01, * * * \mathrm{p}<0.001, * * * * \mathrm{p}<0.0001)$. c-e, After $72 \mathrm{~h}$, the SQAd NAs injected animals showed a complete recovery of their hindlimbs, in accordance with the absence of visible traumatic area on the cord (e) compared to the trauma group (c) and the adenosine treated group (d). $\mathbf{f}-\mathbf{i}$, After $48 \mathrm{~h}$, the ultrastructure of the myelin configuration of the axons was studied by TEM. For the animals treated with the solution of free adenosine (g) or only squalene NAs (h), the small, medium and large sized myelinated axons presented separations and/or interruption in their myelin configuration $(\rightarrow)$, compared to healthy animals (f). As for the SQAd NAs injected animals (i), the examination of white matter showed myelinated axons with separations in myelin configuration of medium sized and large sized axons, when a few of the medium sized and most of the small sized myelinated axons were found to be ultrastructurally normal $(\rightarrow)$. $\mathbf{j}$, The quantification of the damages on the small myelinated axons showed that the SQAd NAs dramatically reduced the damage score (data are presented as mean $\pm \mathrm{SD}, * * * * \mathrm{p}<0.0001$ ) compared to all groups. 

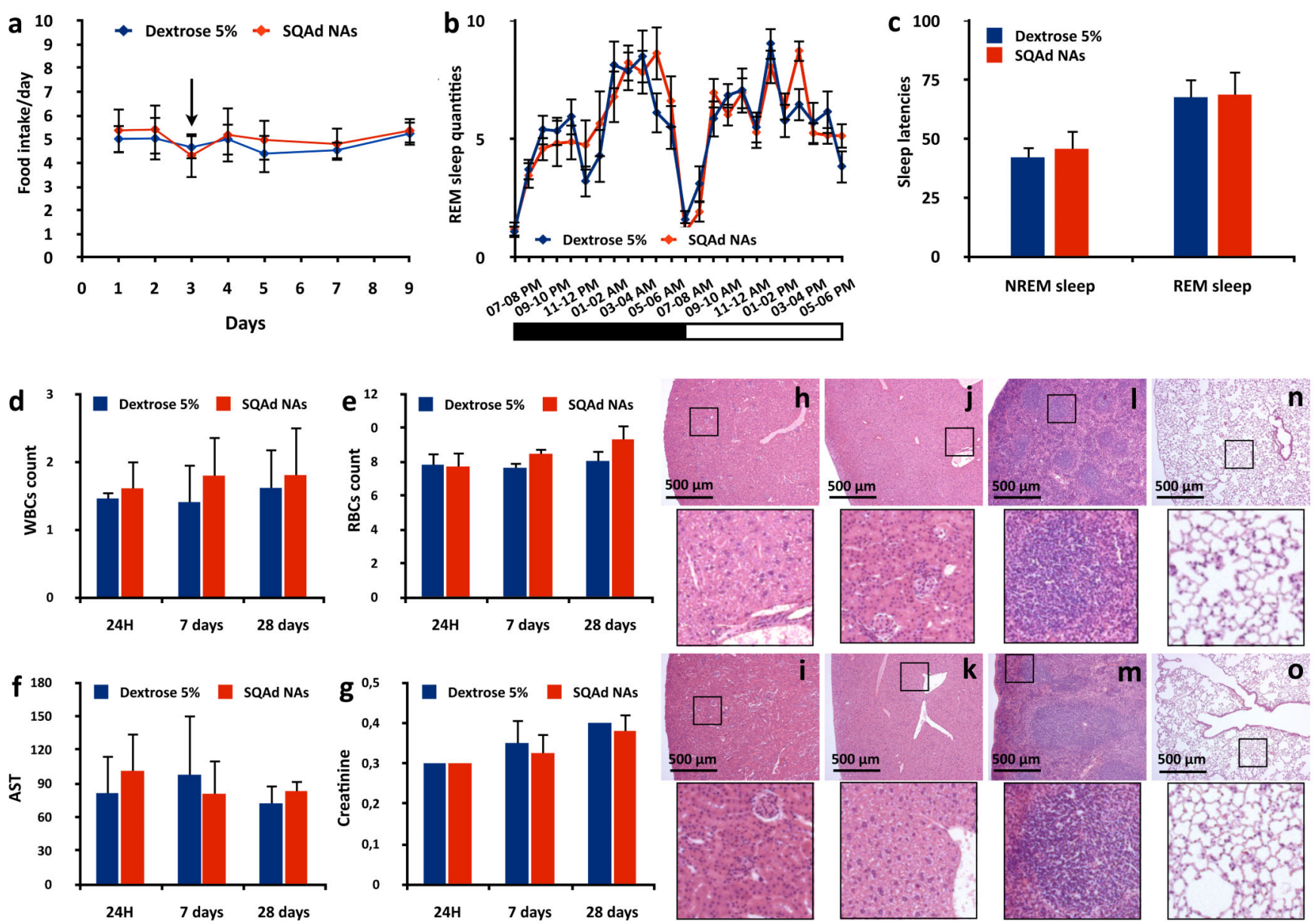

Figure 4. Absence of side effects and systemic toxicity following intravenous injection of SQAd NAs

$\mathbf{a}, \mathbf{b}$, The effect of SQAd NAs on food intake and sleep cycle was assessed in vivo after intravenous injection of either SQAd NAs $(15 \mathrm{mg} / \mathrm{kg})$ or dextrose $5 \%$. Daily food intake of SQAd NAs treated animals was not modified comparatively to controls $(\mathbf{a}, \rightarrow$ indicating the day of SQAd NAs or dextrose $5 \%$ administration, data are presented as mean (gram/day) \pm $\mathrm{SD}, \mathrm{N}=15$ animals/group). The intravenous injection of SQAd NAs did not induce any modification in the REM sleep quantities (b, data are presented as mean (min.) \pm SEM, $\mathrm{N}=$ 15 animals/group, inset showing the alternation of dark and light phases in the recording wardrobe). c, In order to assess if the SQAd NAs could reproduce the sleeping trigger of adenosine, the sleep latencies to NREM and REM sleeps were compared and no differences were observed with dextrose $5 \%$ controls (data are presented as mean (min.) $\pm \mathrm{SEM}, \mathrm{N}=15$ animals/group). Hence, the intravenous injection of SQAd NAs prevents the apparition of any side effects on sleep cycle. d-g, The acute (day 1 and day 7) and long term (day 28) toxicity of the SQAd NAs upon systemic administration were then assessed. SQAd administration did not induce any increase nor decrease of the white (d, data are presented as mean $\left(\times 10^{3} / \mu \mathrm{L}\right) \pm \mathrm{SD}, \mathrm{N}=5$ animals/group) and red blood cells counts (e, data are presented as mean $\left(\times 10^{6} / \mu \mathrm{L}\right) \pm \mathrm{SD}, \mathrm{N}=5$ animals/group). Similarly, no differences in aspartate aminotransferase (f, data are presented as mean (UI/L) $\pm \mathrm{SD}, \mathrm{N}=5$ animals/group) 
and creatinine (g, data are presented as mean $(\mathrm{mg} / \mathrm{dL}) \pm \mathrm{SD}, \mathrm{N}=5$ animals/group) were observed between the animals injected with SQAd NAs and the controls (dextrose 5\%). h-o, The histological analysis of kidneys $(\mathbf{h}, \mathbf{i})$, liver $(\mathbf{j}, \mathbf{k})$, spleen $(\mathbf{l}, \mathbf{m})$ and lungs $(\mathbf{n}, \mathbf{o}), 24 \mathrm{~h}(\mathbf{h}$, $\mathbf{j}, \mathbf{l}, \mathbf{n})$ and 28 days $(\mathbf{i}, \mathbf{k}, \mathbf{m}, \mathbf{o})$ after SQAd NAs intravenous administration $(15 \mathrm{mg} / \mathrm{kg})$, showed no signs of cell or tissue damage. For each picture, inset presents a $5 \times$ magnification of the black square area. 
a

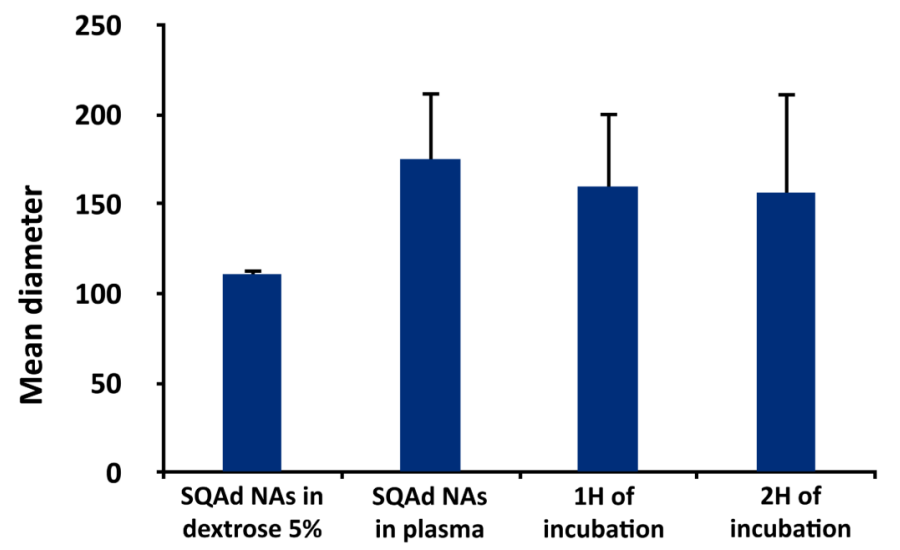

b

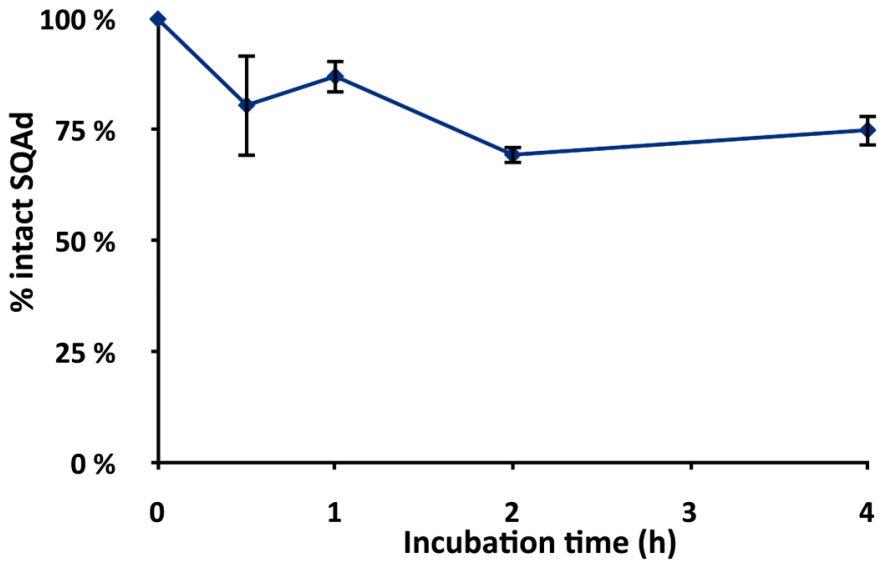

C

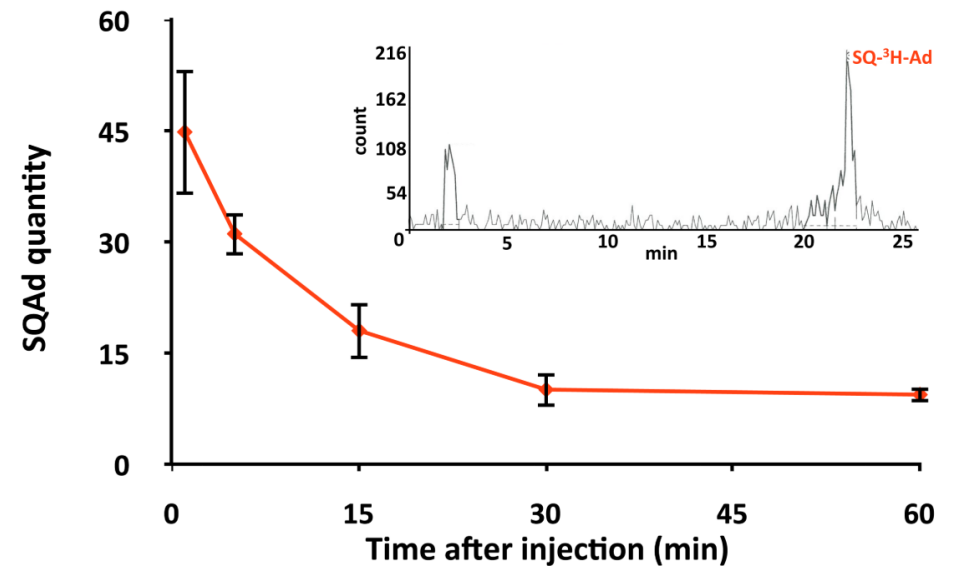

Figure 5. SQAd NAs are a reservoir of adenosine in the systemic circulation a,b, Colloidal stability (a) and chemical stability (b) of the SQAd NAs in mouse plasma at $37^{\circ} \mathrm{C}$. For colloidal stability, the size of the nanoassemblies (data presented as mean \pm SEM, $\mathrm{N}=3$ ) was measured by DLS and chemical stability of SQAd was assessed by HPLC (data presented as mean $\pm \mathrm{SD}, \mathrm{N}=3$ ). $\mathbf{c}$, The plasma concentration of $\mathrm{SQ}-{ }^{3} \mathrm{H}-\mathrm{Ad}$ after intravenous injection of NAs to mice was assessed by radio-HPLC $1 \mathrm{~min}, 5 \mathrm{~min}, 15 \mathrm{~min}, 30 \mathrm{~min}$ and 60 min after administration (data presented as mean (ng of SQAd/mL of plasma) $\pm S D, N=6$ 
animals/group). Representative radio-HPLC chromatogram of plasma collected 5 min after SQ- ${ }^{3} \mathrm{H}-\mathrm{Ad}$ NAs injection is shown in the inset. 

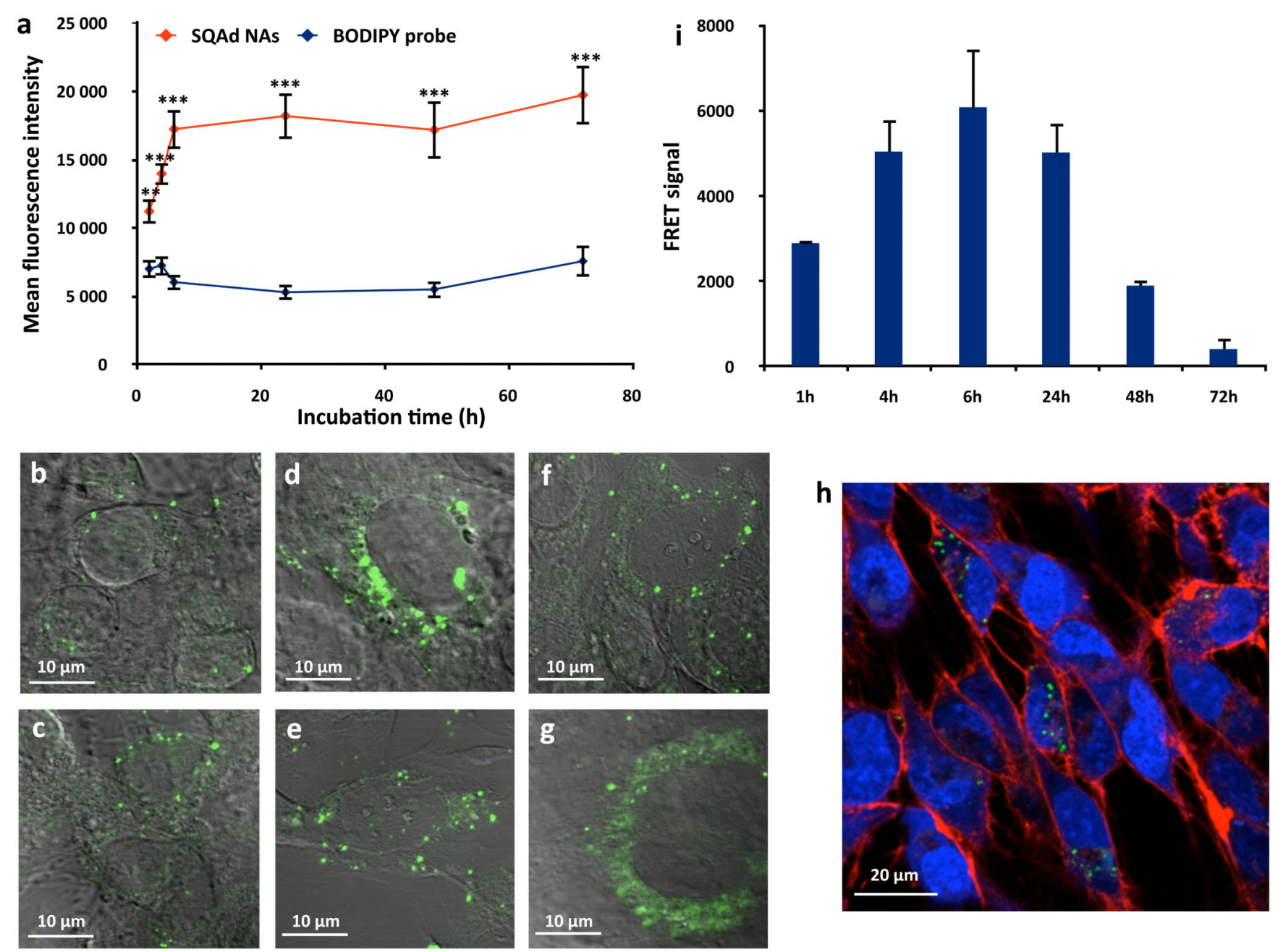

Figure 6. Internalisation of the SQAd nanoassemblies by human cerebral endothelial cells (hCMEC/D3)

a, The cell capture of SQAd NAs by hCMEC/D3 cells was quantified by flow cytometry and compared to the cell internalisation of the fluorescent probe alone $(\mathrm{N}=3$ independent experiments run in duplicate, data are presented as mean (MFI) $\pm \mathrm{SEM}, * * \mathrm{p}<0.01$, $* * * \mathrm{p}<0.001)$. b-g, The effective internalisation of the NAs was confirmed by confocal microscopy after $2 \mathrm{~h}(\mathbf{b}), 4 \mathrm{~h} \mathrm{(c),} 6 \mathrm{~h} \mathrm{(d)}, 24 \mathrm{~h} \mathrm{(e)}, 48 \mathrm{~h}(\mathbf{f})$ and $72 \mathrm{~h}$ (g) of incubation. h, It was observed that the NAs mainly localised in the cell cytoplasm using markers for the nucleus (blue, SYTO61) and for the cellular membrane (red, WGA AlexaFluor 555). i, The internalisation of FRET SQAd NAs showed that the NAs disassembled inside the cells after $6 \mathrm{~h}$ of incubation (data are presented as mean (MFI) \pm SEM, Supplementary Section I.7.). 